\title{
Data Throughputs Using Multiple-Input Multiple-Output (MIMO) Techniques in a Noise-Limited Cellular Environment
}

\author{
Severine Catreux, Peter F. Driessen, and Larry J. Greenstein, Fellow, IEEE
}

\begin{abstract}
We present a general framework to quantify the data throughput capabilities of a wireless communication system when it combines: 1) multiple transmit signals; 2) adaptive modulation for each signal; and 3) adaptive array processing at the receiver. We assume a noise-limited environment, corresponding to either an isolated cell or a multicell system whose out-of-cell interference is small compared with the thermal noise. We focus on the user data throughput, in bits per second/Hertz $(\mathrm{bps} / \mathrm{Hz})$, and its average over multipath fading, which we call the user spectral efficiency. First, an analysis method is developed to find the probability distribution and mean value of the spectral efficiency over the user positions and shadow fadings, both as a function of user distance from its serving base station and averaged over the cell coverage area. We assume fading conditions and receiver processing that lend themselves to closed-form analysis. The resulting formulas are simple and straightforward to compute, and they provide a number of valuable insights. Next, we run Monte Carlo simulations, both to confirm the analysis and to treat cases less amenable to simple analysis.

A key contribution of this paper is a simple formula for the mean spectral efficiency in terms of the propagation exponent, mean signal-to-noise ratio at the cell boundary, number of antennas, and type of coding. Under typical propagation conditions, the mean spectral efficiency using three transmit and three receive antennas ranges from $19.2 \mathrm{bps} / \mathrm{Hz}$ (uncoded) to $26.8 \mathrm{bps} / \mathrm{Hz}$ (ideally coded), highlighting the potential benefits of multiple transmissions combined with adaptive techniques. This is much higher than the spectral efficiencies for a link using a single transmitter and a threefold receive diversity under the same conditions, where the range is from $8.77 \mathrm{bps} / \mathrm{Hz}$ to $11.4 \mathrm{bps} / \mathrm{Hz}$. Moreover, the latter results are not nearly as practical to achieve, as they call for large signal constellations that would be highly vulnerable to impairments.
\end{abstract}

Index Terms-Adaptive modulation, antenna arrays, fading channels, land mobile radio cellular systems, multiple-input multiple-output.

\section{INTRODUCTION}

$\mathbf{M}$ ULTIPLE transmit antennas, adaptive modulation, and adaptive receiver arrays are all targets of current research. A system that combines these three techniques together can provide for very spectrally efficient data transmission, and

Manuscript received March 16, 2000; revised December 19, 2000; accepted May 7, 2001. The editor coordinating the review of this paper and approving it for publication is Dennis L. Goeckel.

S. Catreux is with Iospan Wireless, San Jose, CA 95131 USA.

P. F. Driessen is with the University of Victoria, Victoria, BC V8W 5C3, Canada.

L. J. Greenstein is with AT\&T Labs-Research, Middletown, NJ 07748 USA. Publisher Item Identifier S 1536-1276(02)02093-7. thereby meet the high-speed requirements of future generations of wireless networks.

Adaptive array processing at the receiver has long been used to increase the spectral efficiency of wireless systems, by combating multipath fading [1] or by suppressing interfering signals [2]. More recently, the use of multiple antennas at both the receiver and transmitter [forming a multiple-input multiple-output (MIMO) system] has been shown to increase the spectral efficiency further [3], [4]. Specifically, it was stated that with $n$ transmitting antennas and $m \geq n$ receiving antennas, it is possible to achieve an $n$-fold increase in link capacity, provided that the propagation environment results in significant decorrelation of the complex path gains sampled by the receive array elements. Numerous studies have extended this central result by investigating MIMO capacity under various propagation conditions: line-of-sight (LOS) and Ricean channels in [5]; channels with correlated fading in [6]; and time-varying-channels in [7]. In addition, several implementation techniques have been proposed to make practical the high capacities predicted by information theory. A realizeable architecture of an advanced system is explained in [8]. A simplified approach, called vertical bell labs layered space-time (V-BLAST) is thoroughly described in [9] and compactly presented along with experimental results in [10].

Adaptive modulation belongs to another class of spectrally efficient techniques, referred to as link adaptation, wherein the basic idea is to adapt the transmission parameters (transmitted power, modulation rate, coding rate, spreading factor, etc.) to take the fullest advantage of prevailing channel conditions. The advantage of adaptive modulation combined with a power control scheme has been presented in various contexts, e.g., the single-user case in [11], the multiuser case in [12]. We also note that current proposals for third-generation wireless systems include link adaptation [13], [14].

We assume adaptive modulation is used in conjunction with the MIMO technique, i.e., each transmit signal uses a separately adaptive modulation, matched to the instantaneous channel condition. This is in contrast to V-BLAST, which imposes the same data rate on all transmitters. Our goal is to investigate the theoretical performance of such a system, via both analysis and simulation, and to compare it with more conventional approaches that use receive-diversity only, or no diversity at all. The metric we obtain for a given user is the average, taken over the multipath fading (but not the shadow fading), of the information bit rate divided by the user bandwidth, and is referred to here as the spectral efficiency in bits per second/Hertz (bps/Hz). By means 


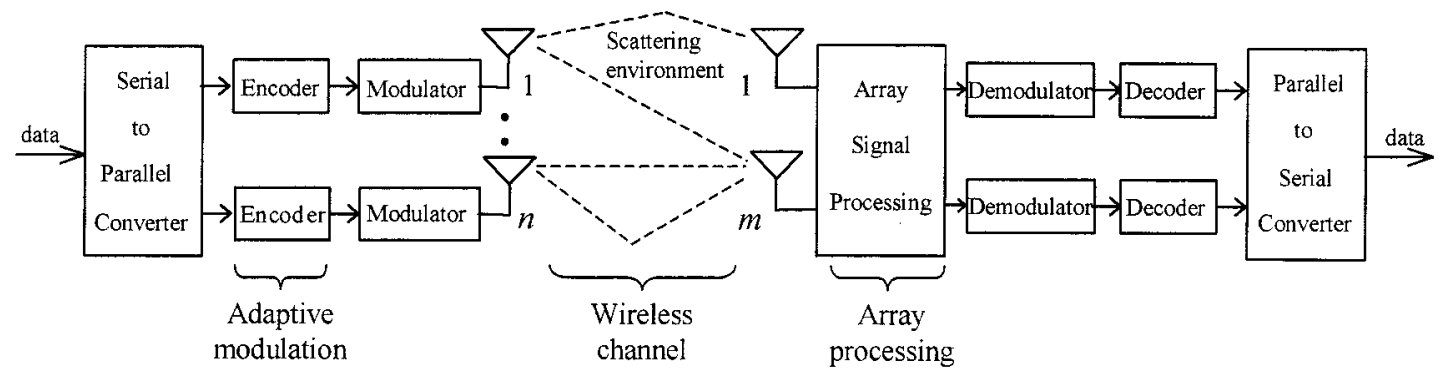

Fig. 1. Model of digital communication system with multiple transmitting and receiving antennas.

of a novel approximation, we are able to bracket the range of this metric over all possible coding approaches, from no coding to the Shannon limit. Assuming fading conditions and receiver processing that lend themselves to closed-form analysis, we derive the probability distribution and mean of this metric, first across users at a distance $d$ from the cell center, and then across all users in the cell.

We consider all links to be noise-limited, meaning either a single-cell environment or a multicell one in which the out-of-cell interference is small compared with the thermal noise. We also assume omni-directional antennas, so that the received signal power is independent of the azimuth of the mobile user (the extension to sectored antennas is straightforward), and a form of minimum mean square error (MMSE) processing at the receiver. We derive an analytical approach that offers valuable insights on the influence of key system and propagation parameters. Then we run Monte Carlo simulations, both to confirm the analysis and to treat cases less amenable to simple analysis. Finally, we summarize our numerical findings and discuss possible extensions of the work.

\section{SYSTEM MODEL}

\section{A. The Radio Link}

A communication system that employs multiple transmitting and receiving antennas can be described as follows (see Fig. 1). A user's bit stream is demultiplexed among several transmitting antennas, each transmitting an independently modulated signal simultaneously and on the same carrier frequency. These signals are received by an antenna array whose sensor outputs are processed such that the original data stream can be recovered.

There are $n m$ radio paths between the $n$ transmit antennas and the $m$ receive antennas. We assume each is complex Gaussian (Rayleigh fading), independent of the others, slow enough to be fixed over a data block, and nondispersive (flat fading). Based on these assumptions, the discrete-time data model for a MIMO system that uses $n$ transmit antennas and $m$ receive antennas in a noise-limited environment can be written as follows:

$$
\boldsymbol{r}=\boldsymbol{H u}+\boldsymbol{\eta}
$$

where $\boldsymbol{r}=\left[r_{1} \ldots r_{m}\right]^{T}$ and $\boldsymbol{u}=\left[u_{1} \ldots u_{n}\right]^{T}$ are the received and transmitted signal vectors at a symbol sampling time. Note that each transmit antenna conveys a distinct bit substream, separately modulated and encoded of equal power $P / n$, i.e., the total transmitted power $P$ is independent of $n ; \eta=\left[\eta_{1} \ldots \eta_{m}\right]^{T}$ is the complex additive white Gaussian noise (AWGN) vector, with statistically independent components of identical power $\sigma_{n}^{2}$ at each of the $m$ receiver branches; and $\boldsymbol{H}$ is the $(m \times n)$ matrix of channel coefficients $\left\{h_{i j}\right\}, 1 \leq i \leq m \quad 1 \leq j \leq n$ where $h_{i j}$ is the complex signal path gain from transmitter $j$ to receiver $i$. This gain is modeled by

$$
h_{i j}=\sqrt{c \frac{1}{d^{\gamma}}} \sqrt{s} \cdot z_{i j}
$$

where $d$ is the base-mobile distance in kilometer, $\gamma$ is the path loss exponent, $c$ is the median of the mean path gain at a reference distance $d=1 \mathrm{~km}, s$ is a log-normal shadow fading variable, where $10-\log s$ is a zero-mean Gaussian random variable (meaning that the median of $s$ is one) with standard deviation $\sigma$, and $z_{i j}$ represents the phasor sum of the multipath scatter components and is a zero-mean unit-variance complex Gaussian random variable. The receiver input signal-to-noise ratio (SNR), averaged over multipath fading, is the same for each branch. This quantity is denoted $\overline{S N R}$, and is a random variable over the shadow fading at a given $d$. The median of this random variable when the mobile is at the maximum $d$ (the apex of the hexagonal cell) is a chosen parameter in our simulations, denoted by $\rho$. Using (2), we can write the median over shadow fading of the multipath-averaged received SNR as

$$
\operatorname{med}(\overline{S N R})=\rho\left(\frac{D}{d}\right)^{\gamma}
$$

where $D$ is the radius of the circle that circumscribes the hexagonal cell.

We consider two alternative schemes for separating the $n$ transmitted signals in the receiver. One scheme linearly combines the received signals using a set of weights that yields the MMSE between the detected data and the true signal samples (MMSE scheme). The second scheme, called ordered successive interference cancellation-MMSE (OSIC-MMSE) is an improved version of MMSE suggested in [9] and [15]. It is a recursive procedure that sequentially detects the different signal components in an optimal order. First, MMSE combining is applied to the received signal vector. Then the substream with the highest output signal-to-interference-plus-noise ratio (SINR) ${ }^{1}$ is detected, and its contribution is subtracted from the total received vector signal. The same process is repeated until all $n$

\footnotetext{
${ }^{1}$ Interference refers, throughout the paper, to residual interference from other substreams.
} 


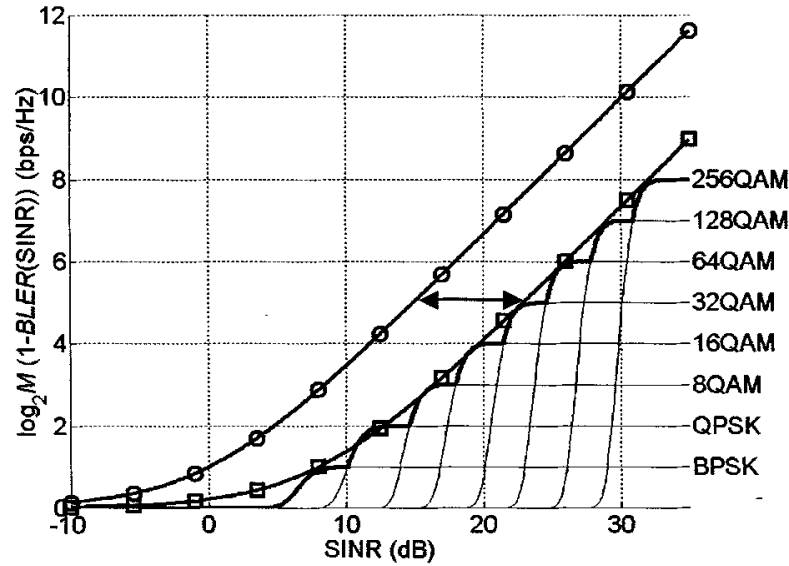

Fig. 2. Throughput (bps/Hz) versus SINR at the output of the combiner. Figure shown for a block length of 500 symbols. $\bigcirc$ ideally coded signals throughput given by Shannon capacity: $\log _{2}(1+S I N R) \square$ uncoded signals throughput given by $\log _{2} M(1-B L E R(S I N R)) \leftrightarrow$ shows the 8-dB shift between the two curves.

substreams are detected. For either scheme, we denote the instantaneous SINR at the $k$ th branch output of the combiner by $Z_{k}, 1 \leq k \leq m$.

\section{B. Adaptive Modulation}

Consider a family of $M$-QAM signal constellations with a symbol period $T_{s}$, where $M$ denotes the number of points in each signal constellation; and assume ideal Nyquist data pulses $\left(\operatorname{sinc}\left[t / T_{s}\right]\right)$ for each constellation. Thus, the channel bandwidth is $W=1 / T_{s}$ and the bit rate is $R=\left(\log _{2} M\right) / T_{s}$. For uncoded $M$-QAM, the attainable normalized throughput in bit per second/Hertz for the $k$ th transmitted substream can be given in terms of the block error rate $(B L E R)$ for block length $L$ as $T_{k}\left(Z_{k}\right)=\log _{2} M_{k} \cdot\left[1-B L E R\left(Z_{k}\right)\right]=$ $\log _{2} M_{k} \cdot\left[1-B E R\left(Z_{k}\right)\right]^{L}$. Here, $B E R$ is the bit-error rate (BER) for an AWGN channel with $M$-QAM modulation and ideal coherent detection; it can be given as a function of $Z_{k}$, corresponding to the $k$ th transmitted substream. This formula assumes perfect error detection, wherein blocks are correctly detected if and only if all bit decisions are error-free. We assume $L$ symbols per block, independent of the signal constellation. An alternative is to keep the number of bits per block fixed, but that would require using different block lengths for different substreams.

Fig. 2 shows a family of curves of $T$ for a given substream (we omit the subscript $k$ for convenience) as a function of the output SINR, for a range of finite values of $M$ such that $M=2^{j}$, $j=1,2, \ldots, J$ where $j$ is the number of bits per symbol. The Shannon capacity, $C(Z)=\log _{2}(1+Z)$, also plotted on Fig. 2, represents an upper bound on the throughput attainable with coding. We observe that the envelope of the $T$-curves is parallel to the Shannon capacity curve, with a fixed offset of about $8 \mathrm{~dB}$. Thus, the envelope can be expressed in a form similar to the Shannon capacity, i.e., $T(Z) \approx \log _{2}\left(1+Z / K_{s}\right)$ where $10 \log K_{s}=8 \mathrm{~dB}$. This approximates the throughput for a given substream when its modulation is adapted, based on the current value of $Z$, so as to maximize throughput. This approximation holds true for a large range of block lengths $L$. A constant gap between the Shannon capacity and the spectral efficiency of $M$-QAM has also been reported for time-invariant channels with intersymbol interference (ISI) and decision-feedback equalization [16], [17], and is further cited in [11], where the spectral efficiency is obtained for a fixed BER.

Finally, the throughput corresponding to a given user in a given block, denoted by $Y$, is the sum of the throughputs corresponding to its $n$ transmitted substreams. Thus

$$
Y=\sum_{k=1}^{n} T_{k}=\sum_{k=1}^{n} \log _{2}\left(1+Z_{k} / K_{s}\right) .
$$

\section{Comments on Our Assumptions and Metrics}

Our aim here is to quantify basic throughput capabilities in a simple way and, to this end, we have made numerous simplifying assumptions. Regarding the channel, we assume independent, flat Rayleigh fading on all $\mathrm{nm}$ transmit-receive paths, with each path gain varying slowly enough to be constant over a data block. Regarding processing, we assume equal power for all $n$ transmitted substreams, with each substream choosing its modulation/coding scheme independently based on current channel conditions, and with no joint detecting of substreams at the receiver. More optimal choices for power allocation and for transmitter and receiver processing are possible, but it is not clear that the benefits would justify the complexity. Regarding implementation, we assume each receiver accurately and quickly informs the transmitter which modulation/coding to use for each substream; that receiver weight adaptation is ideal; that data overhead is negligible; and so on. Practical impairments in all these areas will reduce the actual throughput, but our purpose is to assess and compare theoretically attainable performance with the least complexity and system specificity, and our assumptions serve that purpose.

The average of $Y$ in (4), over multipath fading is the per-user metric which we call the user spectral efficiency $\bar{Y}$. This quantity is a function of user position (distance from its serving base) and shadow fading, so, we seek its cumulative distribution function (CDF) over all users conditioned on a given distance $d$, and denote the average $m_{y}(d)$. The average of $m_{y}(d)$ over the cell, called the mean spectral efficiency $\overline{m_{y}}$, is our primary metric. In a cell with many user channels, this mean closely approximates the total information rate delivered in the cell divided by the total bandwidth.

\section{ANALYTICAL FRAMEWORK}

We now derive an analytical expression for the CDF of the spectral efficiency for users at a specified distance $d$ from the base. We investigate both the ideally coded case (upperbounded by the Shannon limit) and the uncoded case for systems $(1,1)$, $(1, m)$, and $(n, n)$ where the first number indicates the number of transmit antennas (transmitted substreams) and the second one indicates the number of receive antennas. We can represent the user throughput for all of these cases by one general formula based on (4) as follows:

$$
Y=b \sum_{k=1}^{n} \ln \left(1+a Z_{k}\right)
$$


TABLE I

Key Formulas IN COMPUTING SPECTRAL EFFICIENCY FOR SYSTEMS $(1,1),(1, m),(n, n)$

\begin{tabular}{l|c|c|c}
\hline Systems & $(1,1)$ & $\begin{array}{c}(1, m) \\
(\text { with MRC) }\end{array}$ & $\begin{array}{c}(\boldsymbol{n}, \boldsymbol{n}) \\
\text { (with MMSE or ZF) }\end{array}$ \\
\hline User Throughput & \multicolumn{3}{|c|}{$\bar{Y}=n b \int \ln (1+a \cdot Z) p(Z) d Z$} \\
\hline$p(Z)$ & $p(Z)=\frac{1}{\bar{Z}} e^{-Z / \bar{Z}}$ with $\bar{Z}=\mathrm{A}$ & $p(Z)=\frac{Z^{m-1}}{(m-1) !} \frac{1}{\mathrm{~A}^{m}} e^{-Z / \mathrm{A}}$ & $p(Z)=\frac{1}{\bar{Z}} e^{-Z / \bar{Z}}$ with $\bar{Z}=\frac{\mathrm{A}}{n}$ \\
\hline
\end{tabular}

where $b=1 / \ln 2$ and $a=1$ (ideally coded) or $a=K_{s}=$ $1 / 10^{0.8}$ (uncoded). The user spectral efficiency $\bar{Y}$ is obtained by averaging each term of (5) over multipath fading. Since each substream sees statistically identical fading, the average is the same for all of them. Thus

$$
\bar{Y}=n b \int_{0}^{\infty} \ln (1+a Z) \cdot p(Z) d Z
$$

where $p(Z)$ is the probability density function (pdf) of $Z$.

\section{A. Probability Density Function of the Output SNR}

We now consider the fololowing three cases: $(1,1) ;(1,3)$ with maximal ratio combining (MRC); and $(3,3)$ with zero forcing (ZF) combining. In all three cases, there is no residual interference at the output, so in this section, we refer to the output metric $Z$ as the output SNR.

1) System (1,1): The derivation of the pdf of $Z$ in the case of system $(1,1)$ is straightforward. From (1), the data model can be expressed in a scalar notation as $r=h \cdot u+\eta$. Therefore, the SNR at the receiver is

$$
Z=\frac{|h|^{2} P}{\sigma_{n}^{2}}=|h|^{2} \frac{\rho \cdot D^{\gamma}}{c}
$$

where we use the definition of $\rho$ in (3). For a particular user position, the parameters $s$ and $d$ in (2) are fixed; thus, $h$ is equal to a scalar multiplied by $z$ and, therefore, is a complex Gaussian random variable. Its squared magnitude is exponentially distributed and so the pdf of $Z$ is $p(Z)=1 / \bar{Z} e^{-Z / \bar{Z}}, Z \geq 0$. The mean of $Z$ is easily derived as

$\bar{Z}=\frac{\rho \cdot D^{\gamma}}{c} \cdot E\left[|h|^{2}\right]=\frac{\rho \cdot D^{\gamma}}{c} \cdot \frac{c}{d^{\gamma}} \cdot s \cdot E\left[|z|^{2}\right]=\rho\left(\frac{D}{d}\right)^{\gamma} s$.

For later purposes, we define a quantity $A$ as $\bar{Z}$ for the $(1,1)$ case, i.e.,

$$
A=\rho\left(\frac{D}{d}\right)^{\gamma} s
$$

2) $\operatorname{System}(1, m)$ : For system $(1, m)$, the data model is expressed in vector notation as $\boldsymbol{r}=\boldsymbol{h} \cdot u+\boldsymbol{\eta}$, where $u$ is a scalar and $\boldsymbol{r}, \boldsymbol{h}$, and $\boldsymbol{\eta}$ are $m$-dimensional vectors. Assuming an MRC receiver, it is well known that the SNR at the combiner output equals the sum of the input SNRs of the $m$ branches [1]. The output SNR is then equal to

$$
Z=\frac{P \cdot \sum_{i=1}^{m}\left|h_{i}\right|^{2}}{\sigma_{n}^{2}} .
$$

By combining (2), (3), and (7), we can rewrite (8) as $Z=$ $A \sum_{i=1}^{m}\left(x_{i}^{2}+y_{i}^{2}\right)$ where $x_{i}$ and $y_{i}$ are independent Gaussian random variables of equal variance $1 / 2$. Thus, $Z$ is a sum of $2 m$ independent Gaussian random variables, each with variance $A / 2$. The pdf of $Z$ can then immediately be written down as [1]

$$
p(Z)=\frac{Z^{m-1}}{(m-1) !} \frac{1}{A^{m}} e^{-Z / A}, \quad Z \geq 0 .
$$

3) System $(n, n)$ : For system $(n, n)$, the data model is expressed in matrix notation as in (1), where $\boldsymbol{r}, \boldsymbol{u}$, and $\boldsymbol{\eta}$ are $n$-dimensional vectors $\boldsymbol{H}$ and $(n \times n)$ is an matrix. We consider the high SNR case, where the linear MMSE combiner closely resembles the ZF combiner. (We do this to facilitate analysis, and we show later that the results agree closely with simulations for the MMSE combiner.) Under this assumption, the solution weight matrix is the $(n \times n)$ pseudoinverse matrix of $\boldsymbol{H}$ such that $\boldsymbol{W}^{H} \boldsymbol{H}=\boldsymbol{I}^{n \times n}$. The output of this linear filter can be expressed in general as

$$
\boldsymbol{y}=\boldsymbol{W}^{H} \boldsymbol{r}=\boldsymbol{u}+W^{H} \boldsymbol{\eta} .
$$

The postdetection SNR corresponding to the $k$ th substream is $Z_{k}=E\left[\left|u_{k}\right|^{2}\right] /\left[\sigma_{n}^{2}\left\|(W)_{k}\right\|^{2}\right]$ where $(\boldsymbol{W})_{k}$ denotes the $k$ th column of matrix $W$. In order to analyze the distribution of $Z_{k}$, we must determine the statistical properties of the random variable $\omega=1 /\left(\left\|(\boldsymbol{W})_{k}\right\|^{2}\right)$. Because this involves the reciprocal operation, the analysis requires many steps. A solution can be found in [18] for the more general case where the number of receivers can be larger than the number of transmitters. In the Appendix, we present an alternate derivation for the case $m=n$ which, by simple reasoning, shows that $Z_{k}$ is exponentially distributed for all users, with mean value $\bar{Z}_{k}=E\left[Z_{k}\right]=$ $A / n$. This mean output SNR decreases with $n$ because it is a per-substream quantity, and we have assumed the same transmit power $P$ for all cases, divided evenly among the $n$ substreams. In Table I, we summarize the key formulas in computing results for systems $(1,1),(1, m)$, and $(n, n)$.

\section{B. Short-Term Averaged Throughput (Spectral Efficiency)}

1) Systems $(1,1)$ and $(n, n)$ : Since $p(Z)$ has the same general form for both systems $(1,1)$ and $(n, n)$, we treat the two cases together. Thus, the general expression for the short-term averaged throughput at a specified distance $d$, valid for both systems, is obtained via (6) as $\bar{Y}=n b / \bar{Z} \int_{0}^{\infty} \ln (1+a \cdot Z) \cdot e^{-Z / \bar{Z}} d Z$. We can express this more compactly, and in a form that exploits standard computer 
subroutines as follows. We apply the change of variable $x=1+a \cdot Z$ and get

$$
\bar{Y}=\frac{n \cdot b}{\bar{Z} a} e^{1 / \bar{Z} a} \int_{1}^{\infty} \ln x \cdot e^{-x / \bar{Z} a} d x .
$$

We note from [19] that, $\int_{1}^{\infty} \ln x \cdot e^{-\mu x} d x=1 / \mu \operatorname{expint}(\mu)$, $\mu>0$, where expint is the exponential integral function defined as expint $(\mu)=\int_{\mu}^{\infty} e^{-t} / t d t .^{2}$ Thus

$$
\bar{Y}=n \cdot b \cdot e^{\mu} \cdot \operatorname{expint}(\mu)
$$

where

$$
\mu=\frac{1}{\bar{Z} a}=\frac{n}{A a}, \quad \mu>0 .
$$

2) System (1,3): We now find an expression analogous to (12) for the system $(1, m)$. To carry out the analysis, we will actually have to specify a value for $m$, so we present the case of system $(1,3)$.

Combining (6) and (9) for $n=1$, the short-term averaged throughput becomes

$$
\bar{Y}=\frac{b}{A^{m}(m-1) !} \int_{0}^{\infty} \ln (1+a \cdot Z) \cdot Z^{m-1} e^{-Z / A} d Z .
$$

Again, we seek a more compact form and one that exploits standard subroutines. By using (13), $m=3$ and the change of variable, $x=1+a \cdot Z$, it is easy to get

$$
\bar{Y}=\frac{b}{2} \cdot \mu^{3} e^{\mu} \int_{1}^{\infty} \ln x \cdot(x-1)^{2} \cdot e^{-\mu x} d x .
$$

After some algebra, including integration by parts, we get the simple expression

$$
\bar{Y}=\frac{b}{2} \mu^{2} e^{\mu}\left(1-\frac{2}{\mu}+\frac{2}{\mu^{2}}\right) \cdot \operatorname{expint}(\mu)+\frac{b}{2} \mu\left(\frac{3}{\mu}-1\right) .
$$

To summarize, we now have for all three systems $(1,1),(1,3)$, and $(n, n)$ an expression for the short-term average throughput, $\bar{Y}$, in terms of the exponential integral function of $\mu$. As noted, however, we wish to find an even simpler expression for $\bar{Y}$, involving only elementary functions of $\mu$, so that it is easy to manipulate analytically. To do this, we compute and plot (12) and (14) versus the parameter $A$ (contained in $\mu$ ) and find a function that fits the plot points for a wide range of values of $A{ }^{3}$ The function we choose has the general form

$$
\bar{Y}=\alpha \ln (1+\beta A)
$$

where $\alpha$ and $\beta$ are constants that depend on the case being studied (e.g., system size and coding). We show one example of curve fitting in Fig. 3 for the case of system $(1,1)$ and ideally coded signals. The exact curve is given by (12) and the fitting curve is given by (15) with $\alpha=1.4$ and $\beta=0.82$. Similarly, close agreements are found for all cases. Table II summarizes

\footnotetext{
${ }^{2}$ There exists another common definition of the exponential integral function, denoted by $E_{i}(\mu)[19]$. The relationship between $(\mu) E_{i}(\mu)$ and is: $\operatorname{expint}(\mu)=-E_{i}(-\mu), \mu>0$.

${ }^{3}$ For small values of $d, A$ can have a very large magnitude. We choose to fit the curve for values of $A$ anywhere between 0.01 and $2 \cdot 10^{7}$. For example, for $\rho=100$ and $s=1$, this corresponds to a range of $(d / D)$ from 12 to 0.037 .
}

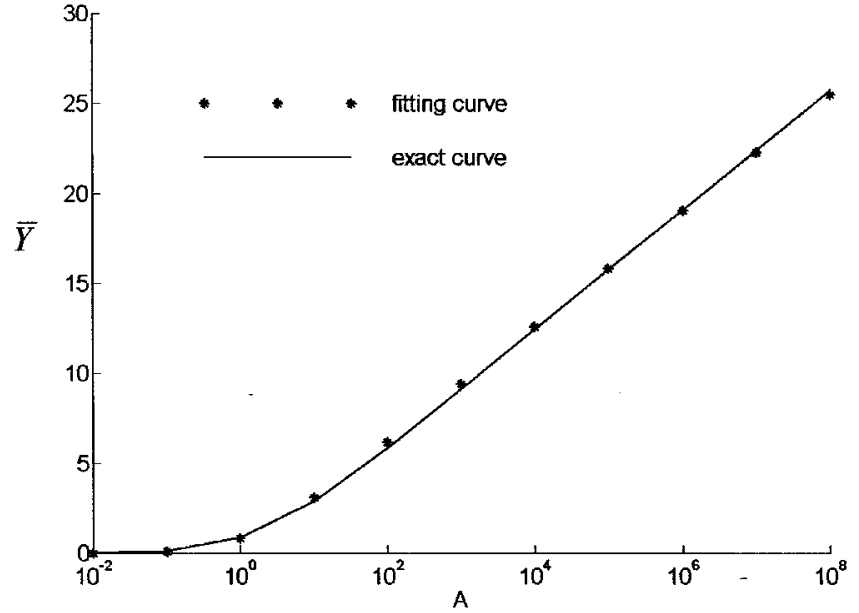

Fig. 3. Short-term averaged throughput as a function of the parameter $A$. Curve fitting for system $(1,1)$ with ideally coded signals.

the exact expression for the short-term averaged throughput and its corresponding approximation.

\section{CDF of Spectral Efficiency Across Users at Distance d}

1) Analytical Derivation: We now derive the CDF of $\bar{Y}$ over the log-normal shadow fading $s$, conditioned on $d$. From (15), we know that $\bar{Y}$ is a monotonic function of $A$. Assuming that the variables $y$ and $a_{0}$ are related by $y=\alpha \ln \left(1+\beta a_{0}\right)$, as in (15), we have

$$
\operatorname{Pr}(\bar{Y} \leq y \mid d)=P_{r}\left(A \leq a_{0} \mid d\right) .
$$

Similarly, $A$ is a monotonic function of $s$ in (7), thus

$$
\operatorname{Pr}\left(A \leq a_{0} \mid d\right)=P_{r}\left(s \leq s_{0} \mid d\right)
$$

where $s_{0}=\left(a_{0} / \rho\right)(d / D)^{\gamma}$. Since $s$ is a log-normal random variable

$$
\operatorname{Pr}\left(s \leq s_{0} \mid d\right)=1-\frac{1}{2} \operatorname{erfc}\left(\frac{10 \log _{10}\left(s_{0}\right)-M_{R}}{\sqrt{2 \sigma^{2}}}\right)
$$

where $M_{R}$ and $\sigma_{2}$ are the mean and variance of $s$ expressed in decibel. Combining (7), and (16)-(18), we get

$$
\operatorname{Pr}(\bar{Y} \leq y \mid d)=1-\frac{1}{2} \operatorname{erfc}\left(\frac{10 \log _{10}\left(\frac{d^{\gamma}\left(e^{y / \alpha}-1\right)}{\rho^{\beta D^{\gamma}}}\right)}{\sqrt{2 \sigma^{2}}}\right) .
$$

We can show that (19) can be well approximated by a Gaussian $\mathrm{CDF}$ of the form

$$
\operatorname{Pr}(\bar{Y} \leq y \mid d)=1-\frac{1}{2} \operatorname{erfc}\left(\frac{y-m_{y}}{\sqrt{2} \sigma_{y}}\right) .
$$

To do this, the term between parentheses in (19) is rewritten as

$$
\frac{10 \ln \left(\frac{d^{\gamma}\left(e^{y / \alpha}-1\right)}{\rho_{\beta} \beta D^{\gamma}}\right)}{\ln (10) \sqrt{2 \sigma^{2}}}=\frac{\ln \left(\frac{d^{\gamma}}{\rho \beta D^{\gamma}}\right)+\ln \left(e^{y / \alpha}-1\right)}{\ln (10) \sqrt{\frac{2 \sigma^{2}}{10}}} .
$$

Next, we make the approximation

$$
\ln \left(e^{y / \alpha}-1\right) \approx \frac{y}{\alpha}
$$


TABLE II

EXACT AND APPROXIMATE EXPRESSIONS FOR $\bar{Y}$ FOR Systems $(1,1),(1,3)$, AND $(n, n)$, AND FOR BOTH IDEALLY CODED AND UNCODED CASES

\begin{tabular}{|c|c|c|c|}
\hline Systems & $\bar{Y}$ Expressions & $a=1$ (ideally coded) & $a=1 / 10^{0.8}$ (uncoded) \\
\hline \multirow[t]{2}{*}{$(1,1)$} & \multicolumn{3}{|c|}{$\bar{Y}=b \cdot e^{\mu} \cdot \operatorname{expint}(\mu)$ with $\mu=\frac{1}{\mathrm{~A} a}$} \\
\hline & $\bar{Y}=\alpha \ln (\beta \mathrm{A}+1)$ (approximation) & $\begin{array}{l}\alpha=1.4=\alpha_{1} \\
\beta=0.82=\beta_{1}\end{array}$ & $\begin{array}{l}\alpha=\alpha_{1} \\
\beta=\beta_{1} / 10^{0.8}\end{array}$ \\
\hline \multirow[t]{2}{*}{$(1,3)$} & \multicolumn{3}{|c|}{$\bar{Y}$ (exact expression): $\quad \bar{Y}=\frac{b}{2} \mu^{2} e^{\mu}\left(1-\frac{2}{\mu}+\frac{2}{\mu^{2}}\right) \cdot \operatorname{expint}(\mu)+\frac{b}{2} \mu\left(\frac{3}{\mu}-1\right)$} \\
\hline & $\bar{Y}=\alpha \ln (\beta \mathrm{A}+1)$ (approximation) & $\begin{array}{l}\alpha=\alpha_{1} \\
\beta=5 \cdot \beta_{1}\end{array}$ & $\begin{array}{l}\alpha=\alpha_{1} \\
\beta=5 \cdot \beta_{1} / 10^{0.8}\end{array}$ \\
\hline \multirow[t]{2}{*}{$(n, n)$} & $\bar{Y}$ (exact expression): & \multicolumn{2}{|c|}{$\bar{Y}=n \cdot b \cdot e^{\mu} \cdot \operatorname{expint}(\mu)$ with $\mu=\frac{n}{\mathrm{~A} a}$} \\
\hline & $\bar{Y}=\alpha \ln (\beta \mathrm{A}+1)$ (approximation) & $\begin{array}{l}\alpha=n \cdot \alpha_{1} \\
\beta=\beta_{1} / n\end{array}$ & $\begin{array}{l}\alpha=n \cdot \alpha_{1} \\
\beta=\beta_{1} /\left(n \cdot 10^{0.8}\right)\end{array}$ \\
\hline
\end{tabular}

which is accurate to within better than $5 \%$ for $y \geq 2.3 \cdot \alpha$. Thus, with the values of $\alpha$ in Table II, this approximation is valid for systems $(1,1)$ and $(1,3)$ for any values of $y \geq 3.2$, and for system $(3,3)$ for any values of $y \geq 10$. We can now match (19) and (20) to find expressions for $m_{y}$ and $\sigma_{y}$

$$
\begin{aligned}
m_{y}(d) & =-\alpha \ln \left(\frac{d^{\gamma}}{\rho \beta D^{\gamma}}\right) \\
\sigma_{y} & =\frac{\sigma \alpha \ln (10)}{10} .
\end{aligned}
$$

Thus, the CDF of $\bar{Y}$ over $s$, conditioned on $d$, can be closely approximated by a Gaussian distribution whose mean and standard deviation are given by (22) and (23). We see that the mean of the distribution does not depend on $\sigma$, that the standard deviation depends on it linearly, and that the mean is a simple function of $d$ and of several system and propagation parameters.

2) Comparison With Simulation Results: In addition to the analysis methodology, we have developed a general-purpose system-level simulation platform, both to confirm the analysis and to treat cases less amenable to simple analysis. Our basic simulation approach is as follows. We generate uniformly distributed random positions of the mobile on a circle of radius $d$ within the cell, and we compute the complex path gains to the serving base, following the channel model given in (2). We use the following parameters values: path loss exponent $\gamma=3.7$, log-normal shadow fading standard deviation $\sigma=8 \mathrm{~dB}$, and the median of $\overline{S N R}$ at the cell extremity is $\rho=100(20 \mathrm{~dB})$. Given the array processing scheme, we compute the output SINR for each transmitted signal from the serving base. We adapt each substream's modulation rate according to the corresponding output SINR and we compute the user throughput for each substream. We then sum the throughput over all $n$ transmitted substreams, and average over the multipath fadings. By repeating this procedure over 2000 random positions/sets of path gains, we find the probability distribution of the user spectral efficiency $\bar{Y}$ over the population of user locations at a distance $d$ from the base. We did this experiment for different values of distance $d$ (normalized to the cell radius), for different systems sizes, and for both ideally coded and uncoded signals. For the (3,3) case, we considered both MMSE and OSIC-MMSE combining, as discussed in Section II-A.

The comparison between simulation and analysis [shown for two cases in Fig. 4(a) and (b)] displays a close agreement. Note that for the analysis, we used the Gaussian approximation in (20). Fig. 4(a) shows the best match and Fig. 4(b) shows the poorest match. The accuracy for every other case treated lies in between. The divergence between simulation and analysis noted in Fig. 4(b) brings us to conclude that, in situations where the spectral efficiency is small, it is no longer Gauss-distributed and, thus, its distribution has to be modeled by the exact distribution given in (19). Note that this latter analytical expression is valid for all values of $y$; it was found to match the simulations almost exactly and, hence, is not plotted.

\section{CDF of Spectral Efficiency Over the Cell}

In Section III-C, we derived the CDF of the spectral efficiency $Y$ at a specific distance $d$. The last step of the analysis is to average this conditional CDF over all user locations and, thereby, obtain the CDF of the spectral efficiency over the cell. A minor complication is that cells are typically modeled as hexagons, a model we followed in conducting simulations over the full cell. To facilitate analysis, we approximate the conventional hexagon cell with maximum distance $D$ by a circle of effective radius $D_{e f}$, defined such that the areas of the hexagon and the circle are the same. It is easy to show that $D_{\text {eff }} / D=\sqrt{3 \sqrt{3} / 2 \pi} \approx 0.91$. Assuming that users are uniformly distributed over a circular area of radius $D_{e f}$, the pdf of $d$ for a randomly selected user location is $p(d)=2 d / D_{e f}^{2}, 0 \leq d \leq D_{e f}$. Thus, the CDF of the averaged throughput over the cell can be obtained from $P_{r}(\bar{Y} \leq y)=\int_{0}^{D_{e f}} P_{r}(\bar{Y} \leq y \mid x) \cdot p(x) d x$, where we applied a change of variable $(d \rightarrow x)$ for notational clarity. We compute this integral numerically and show the results in Fig. 5, where 


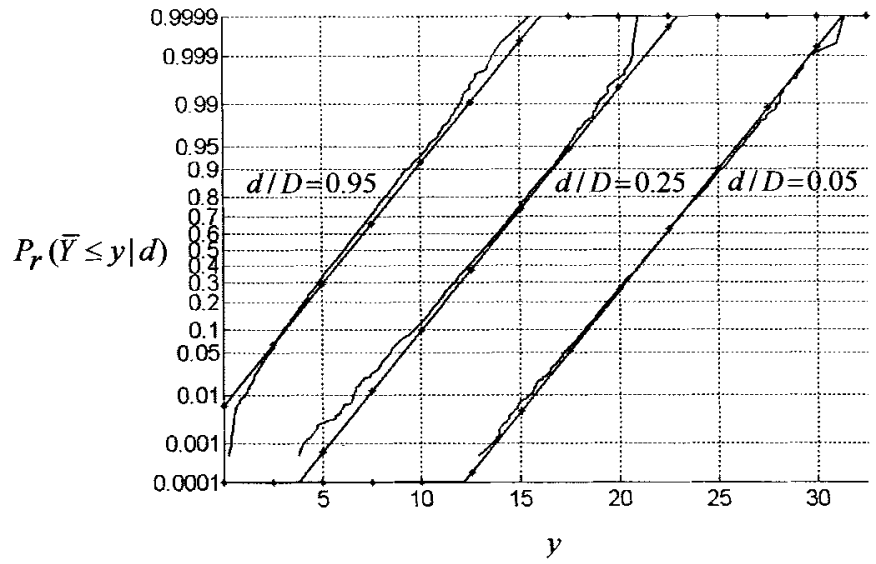

(a)

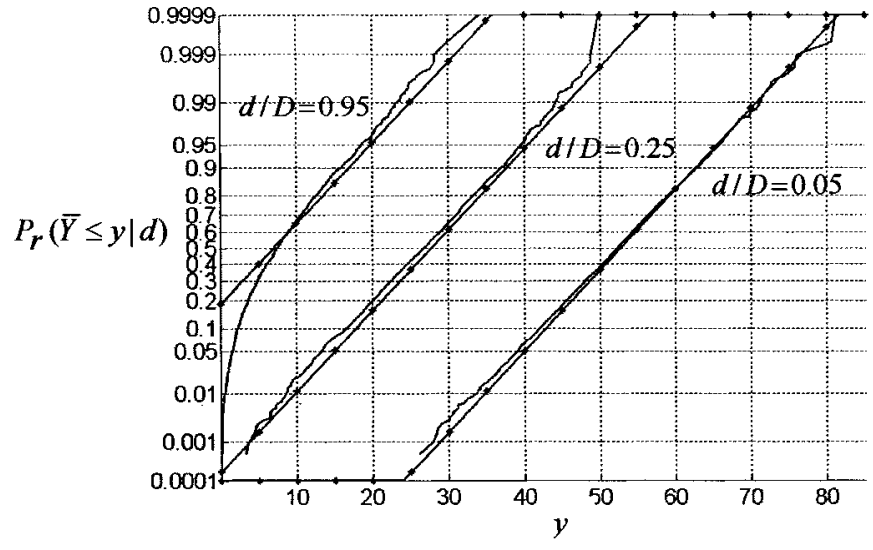

(b)

Fig. 4. (a) Comparison of the $\mathrm{CDF}$ of short-term averaged throughput across users, at preselected distances such that $d / D=(0.05,0.25,0.95)$. Case shown corresponds to system $(1,1)$ with ideally coded signals. The curves are plotted on a probability scale, where a straight line denotes a Gaussian distribution. - Simulation*—— Analysis. (b) Comparison of the $\mathrm{CDF}$ of short-term averaged throughput across users, at preselected distances such that $d / D=(0.05,0.25,0.95)$. Case shown corresponds to system $(3,3)$ with uncoded signals. We start seeing a divergence between simulation and analysis for large values of $d$. Indeed, when the users are on the cell boundary, the throughput is smaller than when the users are closer in and, therefore, the values of $y$ are in the range for which the approximation in (21) tends to be erroneous . — : Simulation; *——: Analysis.

we compare the analytical CDF with the simulated CDF. For the particular case displayed here and all others we examined, agreement between analysis and simulation was found to be excellent. The departure of the simulation curve from analysis at the 99th percentile and beyond is due to the limited number of trials, i.e., the simulation is over 1000 users.

Finally, from the Gaussian approximation in (20) with mean (22), we can derive an analytical expression for the mean of the cell-wide distribution. This mean, averaged over all possible values of $d$ is given by the general expression, $\bar{m}_{y}=$ $\int_{0}^{D_{e f}} m_{y}(x) \cdot p(x) d x$. This yields

$$
\begin{aligned}
\bar{m}_{y} & =\alpha\left(\gamma\left(0.5+\ln \left(\frac{D}{D_{e f}}\right)\right)+\ln (\rho \beta)\right) \\
& \approx \alpha(0.6 \gamma+\ln (\rho \beta))
\end{aligned}
$$

showing the influence of various parameters on this mean spectral efficiency. In a system with a large number of user channels,

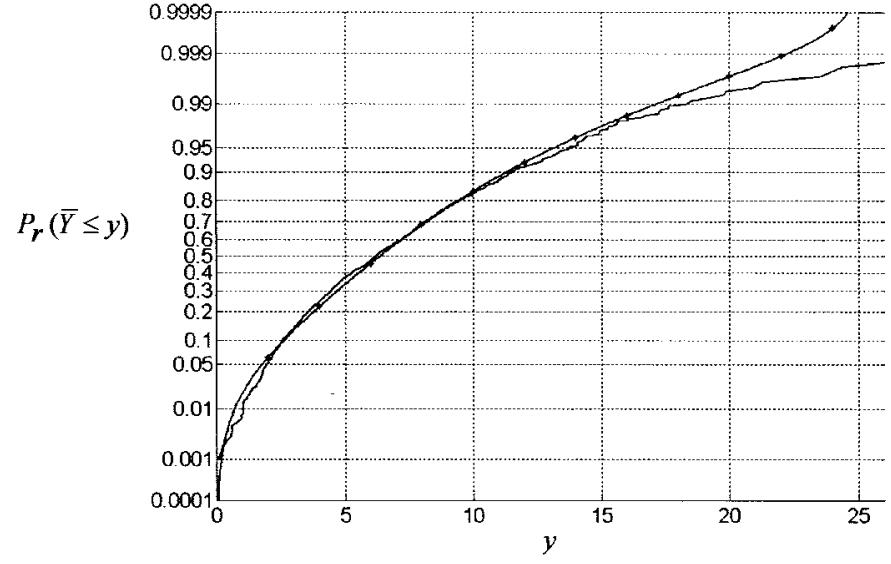

Fig. 5. CDF of throughput across users, over the cell, for system $(1,1)$ with uncoded signals. ——: Simulation; *—— Analysis.

the total throughput per cell will lie in a narrow range about $\bar{m}_{y}$. We, thus, regard $\bar{m}_{y}$ as a useful metric for comparing different designs and channel conditions.

\section{NUMERICAL RESUlTS}

Table III shows the mean spectral efficiency $\bar{m}_{y}$ for various cases. A few key points can be extracted from these results. First, we get a quite close agreement between analysis and simulation, despite three simplifying analytical assumptions: 1) we consider a circle in place of the hexagonal cell; 2) we apply the approximation shown in (21); and 3) we estimate MMSE combining performance by assuming ZF combining. Second, the mean spectral efficiencies attainable with MIMO systems (16.2 to $23.52 \mathrm{bps} / \mathrm{Hz}$ ) go far beyond those of single-input multipleoutput (SIMO) systems (8.77 to $11.4 \mathrm{bps} / \mathrm{Hz}$ ) and single-input single-output (SISO) systems (6.75 to $9.28 \mathrm{bps} / \mathrm{Hz}$ ), which confirms results from [3], [4], and [9]. The large values shown for the SIMO and SISO cases arise from the very large SNRs for users close to the base, which are exploited in our study by assuming correspondingly large constellation sizes. In a practical system, these results would not be realizable because of the vulnerability of such modulations to impairments.

As mentioned earlier, simulations were also used to treat cases less amenable to simple analysis. For example, we modified the channel model in (2) to include a LOS component, whose power is scaled with respect to the scatter component via the Ricean $K$-factor. While the analysis considered a pure scattering environment $(K=0)$, simulations enable us to quantify the effect of higher values of $K$. Furthermore, we used the simulations to investigate the benefits of OSIC-MMSE, described in Section II-A.

Fig. 6 shows the range of mean spectral efficiencies attainable using systems $(1,1),(1,3)$, and $(3,3)$ for the two values $K=0$ and $K=10$. The addition of OSIC to the linear MMSE algorithm improved the mean spectral efficiencies from the range $16.2-23.5 \mathrm{bps} / \mathrm{Hz}$ to the range $19.2-26.8 \mathrm{bps} / \mathrm{Hz}$. This difference can be explained as follows. With the OSIC technique applied to system $(3,3)$, the first (strongest) signal is received with no diversity since the two other substreams must be nulled. The second signal, however, is detected in the presence of only one 
TABLE III

Mean SpeCtral EFFiciency $\bar{m} y$ (BPS/Hz) VIA ANalysis and Simulation

\begin{tabular}{l|l|c|c}
\hline \multicolumn{2}{l|}{ System } & Simulation (bps/Hz) & Analysis (bps/Hz) \\
\hline \multirow{2}{*}{ SISO system (1,1) } & ideally coded & 9.28 & 9.25 \\
\cline { 2 - 4 } & uncoded & 6.75 & 6.67 \\
\hline \multirow{2}{*}{ SIMO system (1,3) } & ideally coded & 11.4 & 11.5 \\
\cline { 2 - 4 } & uncoded & 8.77 & 8.93 \\
\hline \multirow{2}{*}{$\begin{array}{l}\text { MIMO system } \\
\text { (with MMSE) }\end{array}$} & ideally coded & 23.52 & 23.14 \\
\cline { 2 - 4 } & uncoded & 16.2 & 15.4 \\
\hline
\end{tabular}

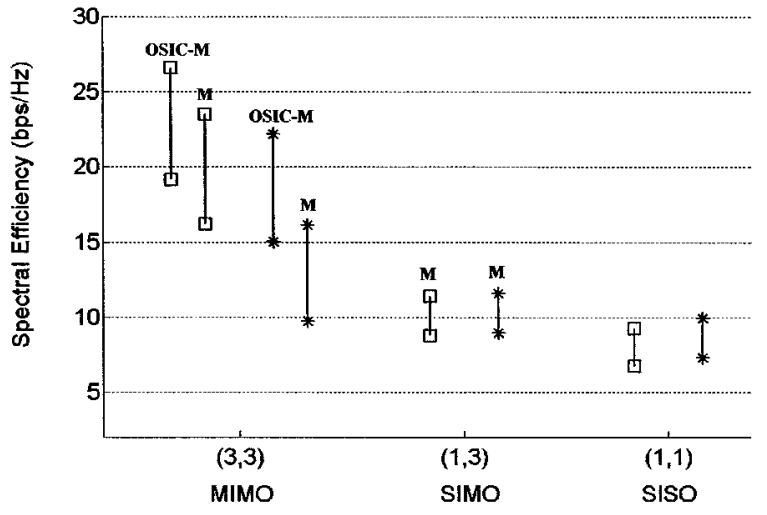

Fig. 6. Mean spectral efficiency (bps/Hz) for systems $(1,1),(1,3)$, and $(3,3)$ in a single-cell environment, downlink, no power control, $\sigma=8 \mathrm{~dB}, \rho=$ $20 \mathrm{~dB}$. Each vertical bar represents the range of spectral efficiencies obtainable between the case of uncoded signals (lower end) and that of ideally coded signals (upper end). The output SINR for system $(1,3)$ was computed using MMSE combining (equivalent to MRC when there are no interferers), and the output SINR for system $(3,3)$ was obtained for two types of combining, i.e., MMSE and OSIC-MMSE. $\square: K=0$. *: $K=10$. M: MMSE combining, OSIC-M OSIC-MMSE combining.

other signal and is, therefore, received with dual-diversity. Finally, the last (weakest) signal is detected with diversity of order 3 , since the two prior detected signals have been cancelled out. With MMSE, each signal is detected in the presence of all the others, and the nulling-out of other signals results in reduced diversity benefits, thus, reduced capacity.

Finally, the influence of $K$ is noticeable for MIMO systems because it affects the correlation among the path gains. As $K$ increases from zero to ten, the throughput of system $(3,3)$ drops by around 20\% for OSIC-MMSE, and by around 40\% for MMSE. Indeed, for closely spaced antennas in an LOS environment, the path gains become highly correlated and, as a result, the MIMO capacity is reduced. Note that this issue was addressed in [5], where a new cell geometry was defined to preserve the separation among paths in the LOS case.

\section{CONCLUSION}

We presented a general framework for studying the performance of MIMO systems in a noise-limited cellular environment. Specifically, we studied the throughput performance that may be achieved by combining multiple transmit signals, adaptive modulation, and adaptive array processing at the receiver. An analysis method was developed to find the probability distri- bution and mean value of the spectral efficiency $(\mathrm{bps} / \mathrm{Hz})$ over the user positions, both within a cell coverage area and as a function of user distance from its serving base station. A key contribution of this paper is a simple formula (24) for the mean spectral efficiency in terms of the propagation exponent, median received SNR at the cell boundary, system size $(n, m)$, and coding type (uncoded or ideally coded). Our results show spectral efficiencies in the range $16-24 \mathrm{bps} / \mathrm{Hz}$ for a MIMO system $(3,3)$ having a median received SNR of $20 \mathrm{~dB}$ at the cell boundary and MMSE combining at the receiver.

These enormous spectral efficiencies were obtained for a system with no external interference. In a heavily-loaded cellular environment, there will be significant co-channel interference (CCI) from surrounding cells. Our simulation platform is also applicable to such interference-limited environments and has been used to quantify spectral efficiencies for that case [20], [21].

The results presented here should be regarded as optimistic upper bounds on the performance achievable by practical systems. In addition to CCI, other limiting factors should be examined to make the results more realistic. Among them are finite constellation sizes [21], implementation errors, channel dispersion, time variations, and correlated path gains.

\section{APPENDIX}

We show here that, in an $(n, n)$ system, the postdetection SNR corresponding to the $i$ th substream, $Z_{i}$, is exponentially distributed. From (1) and (10), the $i$ th output of the combiner can be expressed as

$$
y_{i}=\sum_{j=1}^{n} w_{j i}^{*} \cdot h_{j i} \cdot s_{i}+\sum_{j=1}^{n} \sum_{\substack{k=1 \\ k \neq i}}^{n} w_{j i}^{*} \cdot h_{j k} \cdot s_{k}+\sum_{j=1}^{n} w_{j i}^{*} \cdot \eta_{j}
$$

where we have isolated three components corresponding to: 1) the desired signal $s_{i} ; 2$ ) the self-interference signals $s_{k}$; and 3) the noise.

The ZF algorithm computes the weights $w_{j i}^{*}$ such that the contribution of the self-interference signals is nulled. Thus, for all $k \in\{1, \ldots, n \mid k \neq i\}$, we have $(n-1)$ equations of the form

$$
\sum_{j=1}^{n} w_{j i}^{*} \cdot h_{j k}=0, \quad k=1, \ldots, n ; k \neq i
$$


Since, for given $i$, there are $n$ values of $w_{j i}$ and only $n-1$ constraints, (A-2), there is one remaining degree of freedom. We use it by specifying that

$$
\sum_{j=1}^{n}\left|w_{j i}\right|^{2}=1
$$

This has the effect of constraining the output noise [last term in (A-1)] to have a mean power of $\overline{|\eta|^{2}}$ which is $\sigma_{n}^{2}$. Now, we have $n$ equations to determine the weights $w_{j i}^{*}, j=1, \ldots, n$ which are functions of all the gains $h_{j k}$ except those for $k=i$. Thus, for given $i$, we have a set of weights $w_{j i}^{*}, j=1, \ldots, n$, that eliminates self-interference, resulting in an output whose instantaneous SNR is

$$
Z_{i}=\frac{\left|\sum_{j=1}^{n} w_{j i}^{*} \cdot h_{j i}\right|^{2} \overline{\left|s_{i}\right|^{2}}}{\sigma_{n}^{2}} .
$$

Since the weights are functions of all the path gains except $h_{j i}$, $j=1, \ldots, n$, the summation term in (A-4) is a sum over $n$ complex Gaussian variates that are independent of $\left\{w_{j i}\right\}$. Therefore, the summation term conditioned on $\left\{w_{j i}\right\}$ is itself complex Gaussian, and the conditional pdf of its squared magnitude is thus a one-sided exponential variate. Using (A-3) along with $\overline{\left|s_{i}\right|^{2}}=P / n$, we can give the mean of $Z_{i}$ as

$$
\bar{Z}_{i}=\frac{\frac{P}{n}}{\sigma_{n}^{2}} \overline{|h|^{2}}=\frac{A}{n}
$$

where we have used (2), (3), and (7). Although this result is conditioned on the weights, it is seen to be independent of them. Thus, averaging the conditional pdf of $Z_{i}$ over the joint pdf of the $w$ s yields the result that $Z_{i}$ is unconditionally exponential, with mean given by (A-5).

Extensions of the above line of reasoning can be used to treat the case where the number of receive antennas $m$ exceeds $n$. The general result is that, for $m \geq n$, the output SNR is chisquare-distributed, with $2(m+1-n)$ degrees of freedom.

\section{ACKNOWLEDGMENT}

The authors gratefully acknowledge helpful ideas and comments from M. V. Clark, X. Qiu, L. C. Wang, V. Erceg, and J. H. Winters. They also appreciate the comments of the editor and reviewers.

\section{REFERENCES}

[1] W. C. Jakes, Microwave Mobile Communications. New York: Wiley, 1974.

[2] J. H. Winters, "Optimum combining in digital mobile radio with cochannel interference," IEEE J. Select. Areas Commun., vol. SAC-2, pp. 528-539, July 1984.

[3] _ "On the capacity of radio communication systems with diversity in a Rayleigh fading environment," IEEE J. Select. Areas Commun., vol. SAC-5, pp. 871-878, June 1987.

[4] G. J. Foschini and M. J. Gans, "On limits of wireless communications in a fading environment when using multiple antennas," Wireless Personal Commun., vol. 6, pp. 311-335, Mar. 1998.

[5] P. F. Driessen and G. J. Foschini, "On the capacity formula for multiple-input multiple-output wireless channels: A geometric interpretation,” IEEE Trans. Commun., vol. 47, pp. 173-176, Feb. 1999.
[6] D. Shiu, J. G. Foschini, M. Gans, and J. M. Kahn, "Fading and its Effect on the Capacity of Multi-Element Antenna Systems," in Proc. IEEE Int. Conf. Universal Personal Communication (ICUPC 98), vol. 1, Florence, Italy, 1998, pp. 429-433.

[7] T. L. Marzetta and B. M. Hochwald, "Capacity of a mobile multiple-antenna communication link in Rayleigh flat fading," IEEE Trans. Inform. Theory, vol. 45, pp. 139-157, Jan. 1999.

[8] G. J. Foschini, "Layered space-time architecture for wireless communication in a fading environment when using multiple antennas," Bell Labs Tech. J., vol. 1, pp. 41-59, 1996.

[9] G. J. Foschini, G. D. Golden, R. A. Valenzuela, and P. W. Wolniansky, "Simplified processing for wireless communication at high spectral efficiency," IEEE J. Select. Areas Commun. (Wireless Commununications Series), vol. 17, pp. 1841-1852, Nov. 1999.

[10] P. W. Wolniansky, G. J. Foschini, G. D. Golden, and R. A. Valenzuela, "V-BLAST: an architecture for achieving very high data rates over the rich-scattering wireless channel," in Proc. Int. Symp. Signals, Systems, Electronics (ISSSE-98), Pisa, Italy, Oct. 1998, pp. 295-300.

[11] A. J. Goldsmith and S.-G Chua, "Variable-rate variable-power MQAM for fading channels," IEEE Trans. Commun., vol. 45, pp. 1218-1230, Oct. 1997.

[12] X. Qiu and K. Chawla, "On the performance of adaptive modulation in cellular systems," IEEE Trans. Commun., vol. 47, pp. 884-895, June 1999.

[13] P. Schramm, H. Andreasson, C. Edholm, N. Edvardssona, M. Hook, S. Javerbring, F. Muller, and J. Skold, "Radio interface performance of EDGE, a proposal for enhanced data rates in existing digital cellular systems," in Proc. IEEE 48th Vehicular Technology Conf. (VTC'98), pp. 1064-1068.

[14] P. Bender, P. Black, M. Grob, R. Padovani, N. Sindhushyana, and S. Viterbi, "CDMA/HDR: a bandwidth efficient high speed wireless data service for nomadic users," IEEE Commun. Mag., vol. 38, pp. 70-77, July 2000.

[15] G. D. Golden, G. J. Foschini, R. A. Valenzuela, and P. W. Wolniansky, "Detection algorithm and initial laboratory results using the V-BLAST space-time communication architecture," Electron. Lett., vol. 35, no. 1, pp. 14-15, Jan. 1999.

[16] R. Price, "Non-linearly feedback equalized PAM versus capacity for noisy filter channels," in Proc. IEEE Int. Conf. Communications (ICC'72), Philadelphia, PA, June 1972, pp. 22-12-22-17.

[17] M. V. Eyuboglu, "Detection of coded modulation signals on linear, severely distorted channels using decision feedback noise prediction with interleaving," IEEE Trans. Commun., vol. 36, pp. 401-409, Apr. 1988

[18] J. H. Winters, J. Salz, and R. D. Gitlin, "The impact of antenna diversity on the capacity of wireless communication systems," IEEE Trans. Commun., vol. 42, no. 2/3/4, pp. 1740-1751, Feb./Mar./Apr. 1994.

[19] I. S. Gradshteyn and I. M. Ryzhik, Tables of Integrals; Series and Products, 4th ed. New York: Academic, 1965.

[20] S. Catreux, P. F. Driessen, and L. J. Greenstein, "Simulation results for an interference-limited multiple-input multiple-output cellular system," IEEE Commun. Lett., vol. 4, pp. 334-336, Dec. 2000.

[21] — "Attainable throughput of an interference-limited multiple-input multiple-output (MIMO) cellular system," IEEE Trans. Commun. Lett., vol. 49, pp. 1307-1311, Aug. 2001.

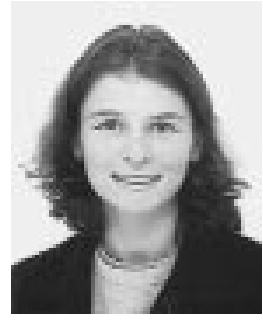

Severine Catreux received the M.Sc. and Ph.D degrees in electrical engineering from National Institute of Applied Sciences (INSA), Rennes, France, in 1996 and 2000, respectively. Between 1996 and 1999, she was at the University of Victoria, BC, Canada, where she studied array signal processing techniques for digital radio communications systems. From April 1999 to early 2000, she completed her doctoral studies in the Wireless Communications Research Department of AT\&T Labs Research, with emphasis on the data throughputs attainable by multiple-input multiple-output (MIMO) systems.

In April 2000, she joined Iospan Wireless Inc., San Jose, CA, a start-up company developing a high-speed broadband fixed wireless system using smart antennas. Her research interests are in the area of adaptive signal processing for digital communications, wireless MIMO systems, and other cutting-edge technologies for future generations of wireless systems. 


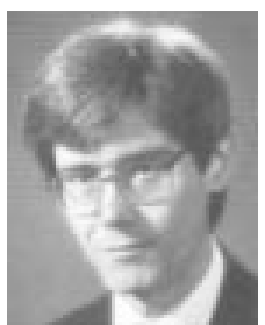

Peter F. Driessen received the Ph.D. degree in electrical engineering from the University of British Columbia, Vancouver, BC, Canada, in 1981.

He has worked with various companies in Vancouver on several projects related to wireless data transmission and modem chip design. Since 1986, he has been at the University of Victoria, Victoria, BC, Canada, where he is now Associate Professor in the Department of Electrical and Computer Engineering. He was on sabbatical leave at AT\&T Bell Laboratories, Holmdel, NJ, during the academic year 1992-1993, and at AT\&T Laboratories-Research, Red Bank, NJ, during the academic year 1999-2000. His research interests are in aspects of wireless communications systems, audio signal processing and streaming multimedia over packet networks. He has served as an editor for IEEE Personal Communications Magazine from 1997 to 1999 and as an editor for IEEE JOURNAL on SElECted AREAS in COMmunications Wireless Communications Series (now IEEE TRANSACTIONS ON WIRELESS COMMUNICATIONS) from 1999 to the present.

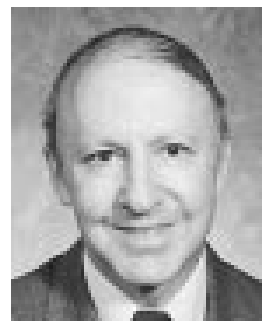

Larry J. Greenstein (M'59-M'67-SM'80-F87) received the B.S., M.S., and Ph.D degrees in electrical engineering from the Illinois Institute of Technology, Chicago, in 1958, 1961, and 1967, respectively.

From 1958 to 1970 , he was with IIT Research Institute, working primarily in the areas of radio-frequency interference and anti-clutter airborne radar. He joined Bell Laboratories in 1970, where he conducted research in communications satellites, microwave digital radio, lightwave transmission, and wireless communications. He is currently a Technology Leader at AT\&T Labs-Research in Middletown, NJ. He co-edited Microwave Digital Radio and has been a guest editor, senior editor, an editorial board member for numerous publications. His areas of concentration in the wireless field include measurement-based propagation modeling, microcell system design and analysis, diversity and equalization, and system performance analysis and optimization.

Dr. Greenstein has won two best paper awards and is an AT\&T Fellow. 